\title{
The efficacy of therapeutic apheresis in patients with refractory neuromyelitis optica spectrum disorders: a single-center retrospective study
}

\author{
Leiying Zhang ${ }^{1 \#}$, Yuan Zhuang ${ }^{1 \#}$, Xiaomin Liu ${ }^{1 \#}$, Quangang $\mathrm{Xu}^{2}$, Lingling Zhou ${ }^{1}$, Liyang Zou ${ }^{1}$, \\ Ying Jiang ${ }^{1}$, Jing Tian $^{1}$, Huan Yao ${ }^{1}$, Hongxu Chi ${ }^{1}$, Xuede Qiu ${ }^{1}$, Tianxin Yang ${ }^{1}$, Deqing Wang ${ }^{1}$, Yang Yu ${ }^{1}$ \\ ${ }^{1}$ Department of Blood Transfusion Medicine, the First Medical Center, Chinese PLA General Hospital, Beijing, China; ${ }^{2}$ Department of \\ Ophthalmology, Chinese PLA General Hospital, Beijing, China \\ Contributions: (I) Conception and design: Y Yu, Y Zhuang; (II) Administrative support: D Wang; (III) Provision of study materials or patients: Q Xu; \\ (IV) Collection and assembly of data: X Liu; (V) Data analysis and interpretation: L Zhang; (VI) Manuscript writing: All authors; (VII) Final approval \\ of manuscript: All authors. \\ \#These authors contributed equally to this work. \\ Correspondence to: Yang Yu; Deqing Wang. Department of Blood Transfusion Medicine, the First Medical Center, Chinese PLA General Hospital, \\ Beijing, China. Email: yuyangpla301@163.com; deqingw@vip.sina.com.
}

Background Neuromyelitis optica spectrum disorders (NMOSD) are associated with recurrent episodes of optic neuritis and transverse myelitis, often resulting in high attack-related disability. Therapeutic apheresis has been recommended as a second-line treatment for steroid-refractory NMOSD. To assess the efficacy and safety of two apheresis techniques, lymphoplasmapheresis (LPE) and therapeutic plasma exchange (TPE), in refractory NMOSD and to provide a new treatment option for patients with refractory NMOSD.

Methods: This retrospective study examined NMOSD patients who had undergone either LPE or TPE treatment between January 2015 and January 2018. The patients were monitored for improvements in disabilities, incidences of adverse reactions, and safety of the procedure over a one-year follow-up period. The primary outcome measures included changes in the visual outcome scale (VOS) score, the expanded disability status scale (EDSS), and the annualized relapse rate (ARR).

Results: Neurological function and objective response rates were significantly improved in $76.5 \%$ of patients treated with LPE and $83.3 \%$ of patients treated with TPE. There were no significant differences in the two treatment groups $(\mathrm{P}=0.392)$. Similarly, there were no differences in the reduction in the relative relapse rate between the two groups $(\mathrm{P}=0.494)$. Adverse reactions, mostly of mild or moderate intensity, were recorded in $9.3 \%$ of procedures in $38 \%$ of patients. The most commonly observed adverse events (AEs) were similar between the two treatment cohorts.

Conclusions: Patients treated with LPE showed improved neurological function comparable to that reported with TPE treatment. No superiority was shown for either of the apheresis techniques.

Keywords: Therapeutic plasma exchange (TPE); lymphoplasmapheresis (LPE); neuromyelitis optica spectrum disorders (NMOSD); refractory.

Submitted Dec 17, 2020. Accepted for publication Mar 01, 2021.

doi: 10.21037/apm-21-177

View this article at: http://dx.doi.org/10.21037/apm-21-177 


\section{Introduction}

Neuromyelitis optica spectrum disorders (NMOSD) are a heterogeneous group of autoimmune inflammationmediated diseases characterized by demyelination and axonal injury in the central nervous system. Inflammatory lesions mainly affect the optic nerve and spinal cord, in addition to a possible influence on the brain stem and cerebrum $(1,2)$. Serum aquaporin-4 immunoglobulin G antibodies (AQP4$\mathrm{IgG}$ ) play a significant role in the pathogenesis of NMOSD. Acute episodes of NMOSD are currently managed by high-dose intravenous methylprednisolone pulse (IVMP). However, in clinical practice, some patients show poor response to high-dose IVMP or do not tolerate longterm administration of methylprednisolone. Refractory NMOSD occurs when symptoms of acute relapse persist after IVMP administration. Numerous studies have demonstrated that therapeutic plasma exchange (TPE) is an effective treatment in the acute phase of NMOSD (3-9). TPE has been recommended by the American Society for Apheresis (ASFA) for NMOSD patients when IVMP therapy is unsuccessful (10).

In addition to the pathogenetic role of B cells and humoral immunity, activated AQP4-specific T cells are believed to play a critical role in the induction of NMOSD lesions (11). Therefore, theoretically, extracorporeal removal of circulating proinflammatory molecules and activated lymphocytes might be a therapeutic option. Lymphoplasmapheresis (LPE) is a combined technique that integrates both TPE and lymphocytapheresis, resulting in both pathogenic antibody removal and immunoregulation (12). In previous studies, lymphocytapheresis was shown to be efficacious in the treatment of autoimmune disorders including psoriasis vulgaris and multiple sclerosis $(13,14)$. Recently, LPE was reported to be effective in patients with GuillainBarre syndrome by directly removing immunoglobulins, complements, monocytes, and fibrinogen, as well as regulating lymphocyte subsets in the peripheral blood (15). Indeed, LPE has been performed on patients with refractory NMOSD in our hospital since 2015 with positive results.

This study examined the application of LPE in cases of refractory NMOSD. To the best of our knowledge, to date, there have been no published reports regarding the feasibility, effectiveness, or adverse events (AEs) of LPE treatment in patients with NMOSD. The goal of this singlecenter retrospective study was to investigate the treatment benefits and safety profile of LPE and TPE in a similar cohort of patients with refractory NMOSD and provide a reference for effective NMOSD treatment in clinical practice. We present the following article in accordance with the STROBE reporting checklist (available at http://dx.doi.org/10.21037/apm-21-177).

\section{Methods}

\section{Patients}

This was a retrospective single - center study performed in the Chinese People's Liberation Army General Hospital (PLAGH) from January 2015 to January 2018. The medical records of NMOSD patients who underwent either LPE or TPE were reviewed. Patients with complications such as other neurological diseases, patients who were withdrawn from treatment early due to thrombosis, and patients who were anti-AQP4 antibody negative or intolerant to the intervention treatment were excluded from this analysis. Patients with primary NMOSD following the definition suggested by the 2015 International Consensus Diagnostic Criteria were finally enrolled into this study (16).

All acute phase patients received preoperative first-line IVMP therapy and demonstrated poor therapy response rates. Therapeutic apheresis was followed by maintenance treatment consisting of oral prednisone that was tapered accordingly with a treatment course of 5 months and patients were counseled to take azathioprine, mycophenolate mofetil or rituximab for life at standard doses to prevent a recurrence. The expanded disability status scale (EDSS) and visual outcome scale (VOS) were applied to evaluate the response rate of treatment. All procedures performed in this study involving human participants were in accordance with the Declaration of Helsinki (as revised in 2013). This study was been approved by the Ethics Committee of the People's Liberation Army General Hospital. All patients provided signed informed consent for treatment.

\section{Apheresis procedures}

LPE and TPE were performed through peripheral veins using the Fresenius COM.TEC cell separator instrument with version 3.0 software (Fresenius Kabi AG, Bad Homburg vor der Höhe, Germany). During LPE, the thin white cell layer containing monocytes/lymphocytes was separated from the whole blood by procedures that enrich for mononuclear cells (MNCs). TPE was performed based on the lymphocytapheresis procedure. Approximately 
Table 1 Visual outcome scale

\begin{tabular}{ll}
\hline Score & \multicolumn{1}{c}{ Snellen chart (visual acuity) } \\
\hline 0 & $20 / 20-20 / 25$ \\
1 & Scotoma but better than 20/30 \\
2 & Visual acuity between 20/30 and 20/59 \\
3 & Visual acuity between 20/60 and 20/199 \\
4 & Visual acuity between 20/200 and 20/800 \\
5 & Child able to count fingers only \\
6 & Child with light perception \\
7 & No perception of light \\
8 & Unknown or unable to be assessed \\
\hline
\end{tabular}

$(2-3) \times 10^{9}$ lymphocytes were removed per procedure. The treatment course for both TPE and LPE comprised of five procedures every other day. During a TPE session, patients received 1.0-1.2 plasma volume exchanges with $10 \%$ albumin and plasma replacement fluid. Approximately 2,000 to $3,500 \mathrm{~mL}$ of plasma were removed.

Prior to therapeutic apheresis, total plasma volume (TPV) was calculated based on height, weight, and hematocrit (HCT) using the following standard formula: TPV $=0.065$ $\times(1-$ HCT $) \times$ weight $(\mathrm{kg})$. In this study, 1 to $1.5 \mathrm{TPV}$ was removed. Replacement fluid included plasma, 10\% albumin solution, and normal saline, wherein plasma accounted for $70 \%$ of the volume. The selection of replacement fluid was based on the patient's coagulation function. In the apheresis procedures, fluid balance was $100 \%$. Acid citrate dextrose (ACD) solution was used as an anticoagulant, and the anticoagulant ratio of ACD to whole blood was 1:12 to 1:16. All patients received $10 \%$ intravenous calcium gluconate during the procedures. According to the patient's vascular condition, venipuncture or central venous catheterization was selected.

\section{Efficacy of treatment}

The effectiveness of the treatment was quantified using standardized performance scales including the visual outcome scale (VOS) (Table 1) and the expanded disability status scale (EDSS) (17). Severity of impairment in the optic nerve and the spinal cord was rated with a scoring system including VOS and EDSS 48 hours before and after treatment. The best corrected visual acuity $\left(V_{A}\right)$ for both the right and left eye was assessed using the Snellen eye test chart or the international type visual acuity chart. For patients with myelitis lesions, physical disability was assessed using the EDSS. $\Delta \mathrm{V}_{\mathrm{A}}$ and $\triangle \mathrm{EDSS}$ (defined as $\mathrm{V}_{\mathrm{A}}$ score and EDSS score before apheresis minus the score of $\mathrm{V}_{\mathrm{A}}$ and EDSS after apheresis) were compared between the two groups of patients.

Relapse was clinically defined as the occurrence of a new neurological symptom 30 days after a previous relapse and an increase in the EDSS score by at least 1 point $(9,18)$. The annualized relapse rates (ARR $=$ total number of relapses/ disease duration) before the start of therapy and one year after therapy were computed for each group. During the one-year follow-up, visual acuity, stepping ability, and selfcare agency were assessed to determine the frequency of relapse. Furthermore, $\triangle \mathrm{ARR}$ (defined as ARR before apheresis minus ARR in the first year) were assessed in both patient cohorts.

\section{Safety}

Safety outcomes were primarily evaluated based on the reports of adverse reactions and clinical laboratory tests. All patients accepted screening for infectious diseases, hepatitis B surface antigen, hepatitis C virus antibody, and human immunodeficiency virus antibody. Cardiac function and vein conditions were assessed in each patient prior to treatment. Blood cell count, coagulation function, electrolyte levels, ionized calcium levels, and albumin/globulin concentrations were assessed before and after treatment. Electrocardiogram (ECG) and blood pressure were monitored during the procedures. All symptoms of adverse reactions to the apheresis procedure were recorded by the bedside nurse and evaluated by the transfusion physicians.

\section{Statistical analysis}

In this study, measurement data of normal distribution were expressed as mean \pm standard deviation $(\mathrm{SD})$, and independent sample t-tests were used for two-group comparisons, and paired $t$-tests were applied for withingroup comparisons. Data with non-normal distribution were expressed as median and interquartile range $[\mathrm{M}(\mathrm{P} 25, \mathrm{P} 75)]$. Comparison between two groups was performed by Mann-Whitney $U$ test. Wilcoxon rank-sum test was applied for within-group comparison. Enumerated data between the treatment groups were conducted by frequency and proportion. Statistical comparisons between groups for categorical variables were performed by chi- 
Patients with acute course of refractory NMOSD who

underwent either LPE or TPE between 2015 and 2018

$(n=121)$

Excluded:

- the ultimate diagnosis of

autoimmune encephalitis $(n=3)$

- early termination of treatment due to thrombosis $(n=2)$

- anti-AQP4 antibody-negative NMOSDs $(n=4)$

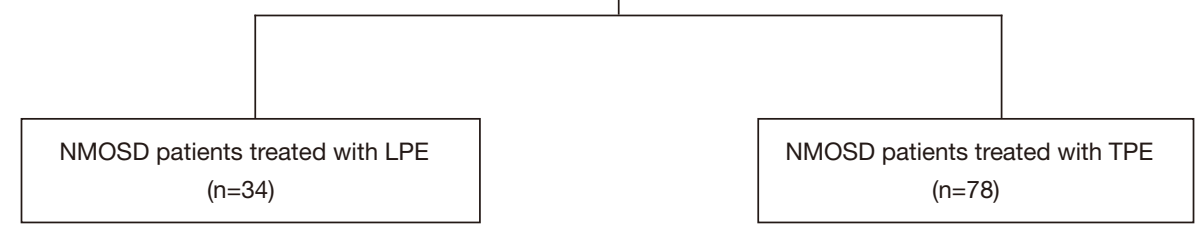

Figure 1 A flow chart showing the patient selection process. NMOSD, neuromyelitis optica spectrum disorders; LPE, lymphoplasmapheresis; TPE, therapeutic plasma exchange; AQP4, aquaporin-4 immunoglobulin G antibodies.

square $\left(\chi^{2}\right)$ test. All statistical tests were performed using SPSS 20.0 (IBM Corp., Armonk, NY, USA) and P values $<0.05$ were considered statistically significant.

\section{Results}

\section{Clinical demographics and characteristics}

During the 3-year study period, a total of 121 patients with NMOSD were admitted and treated with LPE or TPE. There were 3 patients with autoimmune encephalitis, 2 patients with early termination of treatment due to venous thrombosis, and 4 anti-AQP4 antibody-negative NMOSD patients, all of whom were excluded from this analysis (Figure 1).

Finally, 112 eligible patients with refractory NMOSD were enrolled into this retrospective study, and 34 of these patients received LPE, while 78 patients received TPE. The main demographic and clinical characteristics of the two treatment groups are shown in Table 2.

Eighty-two point four percent of the patients in the LPE cohort were female, and the median age was 29 years. Most patients experienced more than 2 relapses, and 50\% of patients relapsed 3 times or more. Acute attacks of optic neuritis were present in $88.2 \%$ of the patients at inclusion, and $11.8 \%$ of patients showed simultaneous presentation of both optic neuritis and severe acute attack myelitis. A total of 6 patients presented with prodromal symptoms preceding an attack of NMOSD. The median intervals from onset of NMOSD to the time of IVMP and therapeutic apheresis was 7 and 28 days, respectively.

The TPE and LPE cohorts were broadly comparable in demographic and most clinical characteristics at inclusion including age of onset, gender, etiology, type of attack at onset, and number of relapses. Before therapeutic apheresis, the $34 \mathrm{LPE}$ patients experienced a mean of 1.5 relapses per year, while the 78 TPE patients showed a mean of 1.6 relapses (Table 3).

\section{Therapeutic evaluation}

The overall rate of improvement in neurological function was $76.5 \%$ (26 of 34 patients) at the end of LPE treatment and $83.3 \%$ (65 of 78 patients) at the end of TPE treatment $(\mathrm{P}=0.392)$. The rate of visual improvement was $66.7 \%$ (28 of 42 eyes) and $71.3 \%$ (72 of 101 eyes) for the LPE group and the TPE group, respectively ( $\mathrm{P}=0.583$, Table 3).

Figure 2 shows the pre- and post-therapy visual acuities in 42 eyes of the $34 \mathrm{LPE}$ patients and 101 eyes of the 78 TPE patients. The visual acuity significantly improved in both groups. The median differences in the visual acuity 
Table 2 The demographic and clinical characteristics of 112 patients with NMOSD

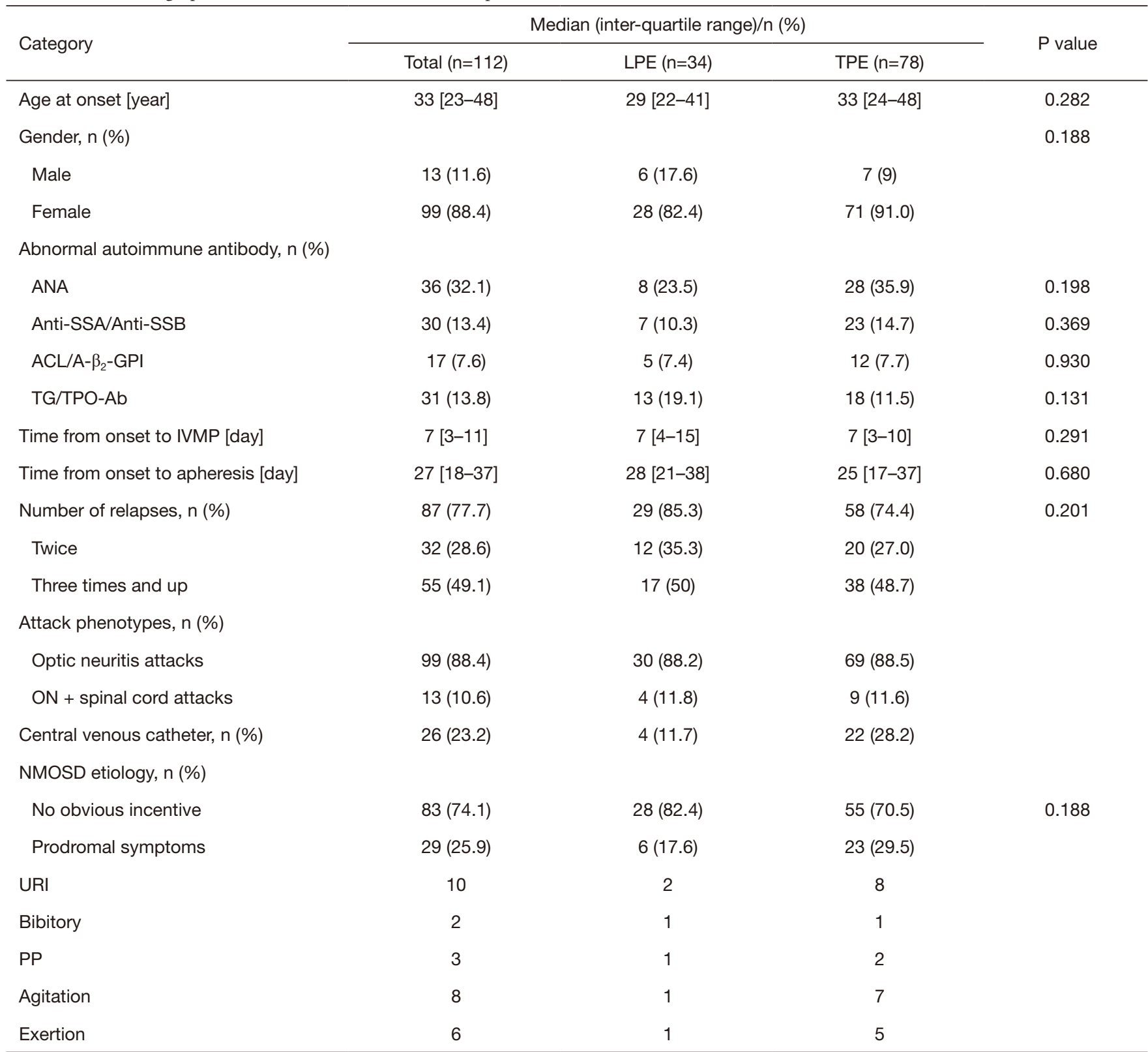

ANA, antinuclear antibody; ACL, anticardiolipin antibody; $A-\beta_{2}-G P I$, anti- $\beta_{2}$-glycoprotein I; RF, rheumatoid factor; TG, anti-thyroglobulin antibodies; TPO, anti-thyroid peroxidase antibodies; AQP4-Ab, aquaporin-4 antibodies; IVMP, intravenous methylprednisolone pulse; ON, optic neuritis; CVC, central venous catheter; NMOSD, neuromyelitis optica spectrum disorders; URI, upper respiratory; PP, post-partum.

scores in the LPE cohort before and after treatment was 5 [4-5] and 4 [2-5], respectively $(\mathrm{P}<0.001$, Figure $2 A)$, and 5 [5-6] and 4 [4-5], respectively for the TPE group $(\mathrm{P}<0.001$, Figure 2B; Table 3).

The changes in the mean EDSS scores and the ARR between the LPE and TPE cohorts are presented in Figures 3 and 4. In the LPE group, the EDSS scores significantly decreased from a mean of 3.4 before treatment to 2.9 after the last treatment $(\mathrm{P}=0.002$, Figure $3 A)$. In the TPE cohort, the EDSS scores also dropped significantly from 4.5 to $3.6(\mathrm{P}=0.011$, Figure $3 B)$.

Before treatment, patients with LPE had a mean of 1.5 relapses per year. In the first year after LPE treatment, 26 patients remained relapse-free, and the mean ARR was 0.6 
Table 3 Treatment efficacy in patients who received LPE and TPE

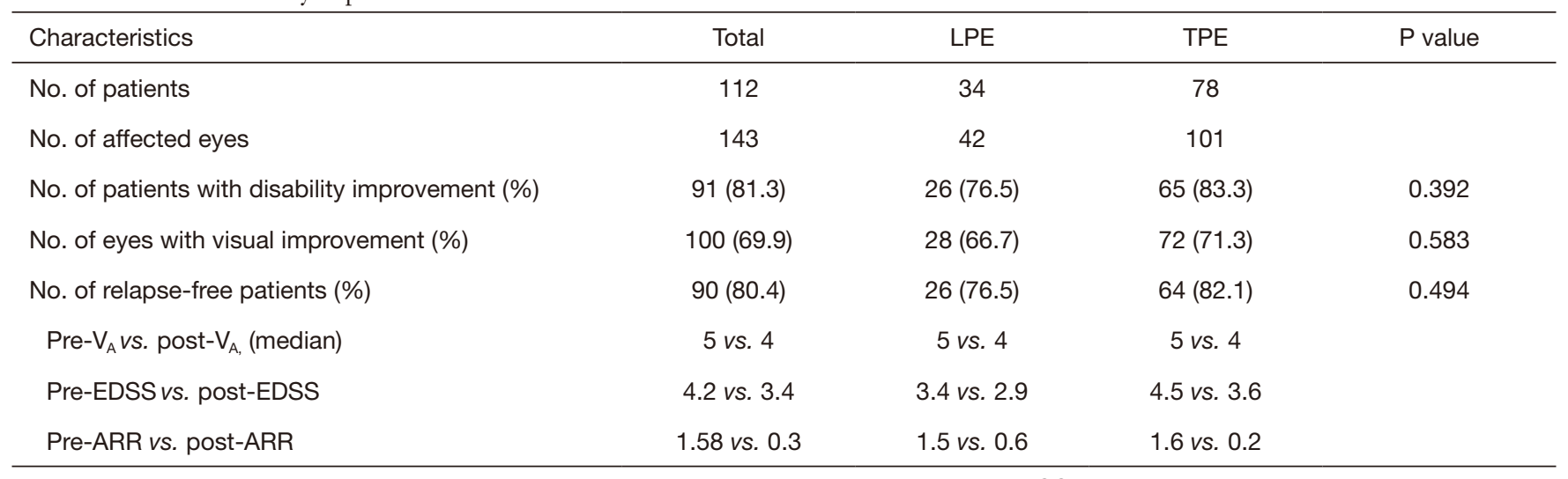

LPE, Iymphoplasmapheresis; TPE, therapeutic plasma exchange; $V_{A}$, visual acuity; EDSS, expanded disability status scale; ARR, annualized relapse rate.

A

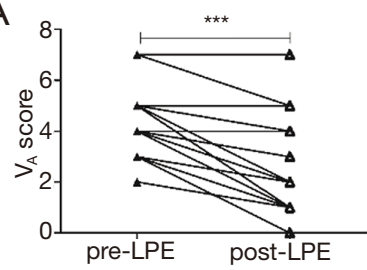

B

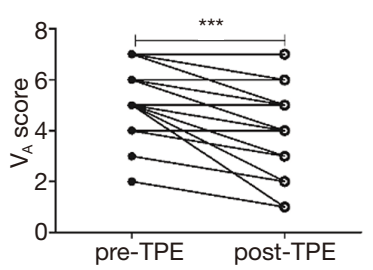

C

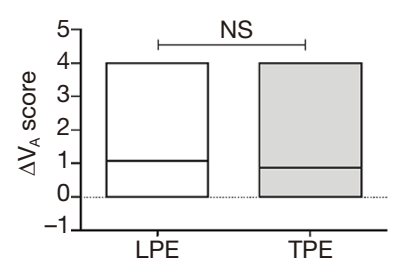

Figure 2 The visual acuity scores pre- and post-therapeutic apheresis in NMOSD patients. (A) The visual acuity scores pre- and posttherapeutic apheresis in NMOSD patients treated with LPE. (B) The visual acuity scores pre- and post-therapeutic apheresis in NMOSD patients treated with TPE. (C) A comparison of the change in visual acuity scores $(\triangle \mathrm{VA})$ in the two treatment groups. ${ }^{* * *} \mathrm{P}<0.001$. NMOSD, neuromyelitis optica spectrum disorders; LPE, lymphoplasmapheresis; TPE, therapeutic plasma exchange; NS, no significant difference.
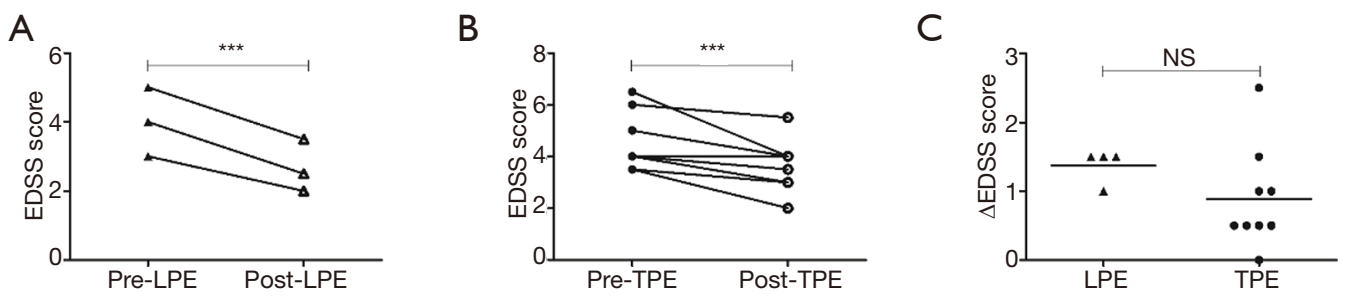

Figure 3 The expanded disability status scale (EDSS) scores pre- and post-therapeutic apheresis in NMOSD patients. (A) The expanded disability status scale (EDSS) scores pre- and post-therapeutic apheresis in NMOSD patients treated with LPE. (B) The expanded disability status scale (EDSS) scores pre- and post-therapeutic apheresis in NMOSD patients treated with TPE. (C) A comparison of the change in the EDSS scores ( $\triangle \mathrm{EDSS}$ ) in the two treatment groups. ${ }^{* *} \mathrm{P}<0.001$. NMOSD, neuromyelitis optica spectrum disorders; LPE, lymphoplasmapheresis; TPE, therapeutic plasma exchange; EDSS, expanded disability status scale; NS, no significant difference.

$(\mathrm{P}<0.001$, Figure $4 A)$. In patients treated with TPE, the ARR decreased from 1.6 to $0.2(\mathrm{P}<0.001$, Figure $4 B)$, and the reduced ARR occurred in 74 patients $(95 \%), 64$ of whom were relapse-free. The primary end points of the study including visual acuity score, EDSS score, and ARR did not significantly differ between the LPE and TPE cohorts
$(\mathrm{P}=0.421$, Figure $2 C ; \mathrm{P}=0.236$, Figure $3 C ; \mathrm{P}=0.117$, Figure $4 C$ ).

\section{Feasibility and safety}

The overall incidence of AEs was similar in the LPE cohort $(27 / 247,10.9 \%)$ and the TPE cohort $(32 / 388,8.2 \%)$. 
A

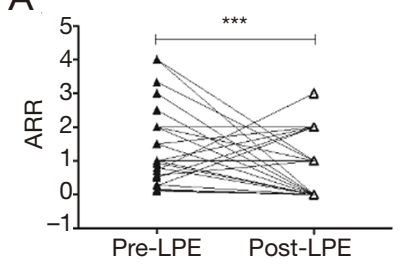

B

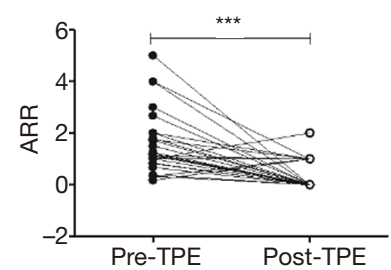

C

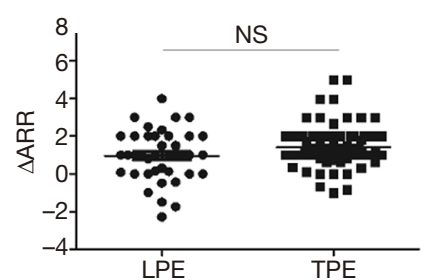

Figure 4 The annualized relapse rate (ARR) pre- and post-therapeutic apheresis in NMOSD patients. (A) The annualized relapse rate (ARR) pre- and post-therapeutic apheresis in NMOSD patients treated with LPE. (B) The annualized relapse rate (ARR) pre- and post-therapeutic apheresis in NMOSD patients treated with TPE. (C) A comparison of the change in the annualized relapse rate ( $\triangle \mathrm{ARR})$ in the two treatment groups. ${ }^{* *} \mathrm{P}<0.001$. ARR, annualized relapse rate; NMOSD, neuromyelitis optica spectrum disorders; LPE, lymphoplasmapheresis; TPE, therapeutic plasma exchange; NS, no significant difference.

Table 4 AEs by severity, type, and frequency for the 112 patients with NMOSD who were treated with LPE and TPE

\begin{tabular}{lccc}
\hline Characteristics & Total & LPE & TPE \\
\hline No. of patients & 112 & 34 & 78 \\
No. of procedures & 635 & 247 & 388 \\
No. of people who incurred AEs & 43 & 19 & 24 \\
Frequency and type of AEs, $n$ (\%) & $59(9.3)$ & $27(10.9)$ & $32(8.2)$ \\
Allergic reactions grade 1 & $43(6.8)$ & $18(7.3)$ & $25(6.4)$ \\
Citrate toxicity grade 1-2 & $5(0.8)$ & $4(1.6)$ & $1(0.3)$ \\
Hematoma grade 1 & $1(0.2)$ & $1(0.4)$ & 0.256 \\
Catheter-related thrombosis grade 1-2 & $4(0.6)$ & 0 & $4(1.0)$ \\
Vasovagal reactions grade 2 & $6(0.9)$ & $4(1.6)$ & $2(0.5)$
\end{tabular}

$\mathrm{AE}$, adverse events; NMOSD, neuromyelitis optica spectrum disorders; LPE, lymphoplasmapheresis; TPE, therapeutic plasma exchange.

A total of 112 patients were treated with 635 sessions of therapeutic apheresis and a total of 59 (27 in the LPE group and 32 in the TPE group) AEs were recorded. All AEs were mild to moderate in severity. No patients experienced severe adverse reactions such as mucosal swelling (angioedema), dyspnea, hypoxia, or shock. As shown in Table 4, the most common AEs were allergic reactions $(43 / 635,6.8 \%$ of all AEs), followed by vasovagal reactions $(6 / 635,0.9 \%)$, citrate toxicity $(5 / 635,0.8 \%)$, catheter-related thrombosis $(4 / 635$, $0.6 \%)$, and hematoma $(1 / 635,0.2 \%)$.

Table 5 shows the changes in laboratory indicators before and after therapy in the 2 treatment groups. Blood clotting time remained within the normal laboratory reference range throughout the treatment period. The hemoglobin, platelet counts, fibrinogen levels, total protein, and serum albumin, as well as serum calcium levels in both groups decreased significantly after treatment. The LPE cohort had significantly lower serum albumin and platelet counts compared to the TPE cohort.

\section{Discussion}

In this retrospective single-center study, $88.4 \%$ of patients were female and the median age at onset was 33 years (range, 22-38 years). A total of 112 Chinese patients were treated with 635 sessions of therapeutic apheresis. Both LPE and TPE were effective in the treatment of refractory NMOSD. Approximately $81.3 \%$ of the patients in this report achieved satisfactory improvement in neurological function.

TPE treatment can be helpful in the recovery from acute attacks of NMOSD (grade 1B evidence II) (10). Out of the 78 patients receiving TPE treatment, $83.8 \%$ showed disability improvement, which is broadly consistent with the TPE response rates detected in other reports (19-21). Due to cost constraints, relevant tests were not conducted to determine the expression of AQP4-IgG after treatment. 
Table 5 Changes in the laboratory indicators before and after LPE or TPE treatment

\begin{tabular}{|c|c|c|c|c|}
\hline Index & \multicolumn{2}{|c|}{ LPE $(n=34)$} & \multicolumn{2}{|c|}{ TPE $(n=78)$} \\
\hline HGB (g/L) & $129.76 \pm 13.45$ & $124.59 \pm 14.26^{b}$ & $129.37 \pm 14.42$ & $123.64 \pm 14.85^{b}$ \\
\hline PLT (×10\%ㄴ) & $221.50 \pm 50.00$ & $155.79 \pm 44.28^{b}$ & $243.82 \pm 69.60$ & $207.56 \pm 54.28^{\mathrm{bc}}$ \\
\hline FIB (g/L) & $2.55 \pm 0.70$ & $1.92 \pm 0.47^{b}$ & $2.60 \pm 0.76$ & $2.00 \pm 0.39^{b}$ \\
\hline ALB (g) & $39.90 \pm 4.14$ & $34.10 \pm 3.52^{b}$ & $39.93 \pm 3.62$ & $36.12 \pm 2.38^{\mathrm{bc}}$ \\
\hline $\mathrm{Ca}(\mathrm{mmol} / \mathrm{L})$ & $2.20 \pm 0.09$ & $2.12 \pm 0.19^{\mathrm{a}}$ & $2.18 \pm 0.14$ & $2.12 \pm 0.10^{b}$ \\
\hline
\end{tabular}

LPE, lymphoplasmapheresis; TPE, therapeutic plasma exchange; HGB, hemoglobin; PLT, platelet counts; FIB, fibrinogen; TP, total protein; ALB, serum albumin; Ca, serum calcium. ${ }^{a}, \mathrm{P}<0.05 ;{ }^{b}, \mathrm{P}<0.01$ compared to before treatment in the same group; ${ }^{\mathrm{c}}, \mathrm{P}<0.01$ compared with the LPE treatment group after treatment.

There is a high recurrence of NMOSD and this can lead to progressive disability. Within 5 years of disease onset, half of the individuals diagnosed with neuromyelitis optica (NMO) will require the use of a wheelchair or become functionally blind (22). Therefore, prevention of relapse is essential. It is widely accepted that NMOSD is mediated by humoral-immunity and B cells can enhance humoral immune responses and antigen presentation, produce proand anti-inflammatory cytokines, and immunoglobulin generation in the NMO (23). In addition, AQP-4 antibody production requires the help of $\mathrm{T}$ lymphocytes (24). Thus, we speculated that LPE may partially modify the function of the inflammatory network or eliminate immune cell clones non-specifically by removing the peripheral blood lymphocytes. Most importantly, this study demonstrated that LPE might have a beneficial clinical effect in patients with refractory NMOSD. First, LPE improved neurological disability (3.4 vs. $2.9, \mathrm{P}<0.001)$ and significantly reduced the frequency of NMOSD relapses $(1.5$ vs. $0.6, \mathrm{P}<0.001)$. In this study, 26 of 34 patients $(76.5 \%)$ were relapsefree after LPE treatment, and $76.5 \%$ showed improved neurological function. Second, the LPE procedure was feasible and well tolerated, with an AE rate of $10.9 \%$. A study by Kim et al. demonstrated that rituximab therapy resulted in a strong reduction in ARR (2.4 vs. 0.1, $\mathrm{P}<0.001)$ and EDSS score $(4 v s .3, \mathrm{P}<0.001)$ with a median treatment period of 67 months. Of the 100 patients, $70 \%$ were relapse free (25). AEs were experienced by $26 \%$ of patients including infusion-related reactions. However, none required discontinuation of treatment. Another study by Chen et al. reported that almost $72 \%$ of patients treated with tacrolimus were relapse-free (26). Although these studies demonstrated that immunomodulatory and immunosuppressive agents are effective, their efficacy can be limited by frequent adverse effects.

The apheresis technique is a relatively safe procedure with a low incidence of adverse reactions. Different types of AEs have different predisposing factors, mainly related to the type and volumes of replacement fluid, anticoagulant, and vascular access. A total of 59 AEs occurred among 635 procedures $(9.3 \%)$ in this current report. Most AEs reported were of mild-to-moderate intensity and occurred at similar frequencies in both the TPE and LPE cohorts. The most commonly observed AEs were allergic reactions, mainly involving cutaneous symptoms. The frequency of allergic reactions in the LPE and TPE group was $7.3 \%$ $(18 / 247)$ and 6.4\% (25/388), respectively. Most allergic reactions were associated with antibody development in a transfusion recipient specific to the donor protein or the patient's allergic constitution, such as family history of asthma or individual history of hypersensitivity (27). In addition, multiple plasma exchanges can increase the risk of allergic reactions. Currently, antihistamines are most often used in the management of allergic reactions (28). Appropriate normal saline and albumin replacement of plasma can also reduce allergic reactions. In our study, $8 \%$ $(5 / 59)$ of all AEs presented as citrate toxicity of moderate intensity including paresthesia, flushed sensation, nausea, and/or vomiting. Approximately $0.9 \%$ (6/635) of the apheresis procedures resulted in vasovagal reactions, mainly manifesting as pallor, weakness, dizziness, diaphoresis, nausea and/or vomiting, hypotension, and presyncope. Four catheter-related thrombotic events $(0.6 \%)$ occurred in 26 patients with indwelling central venous catheters. No 
complications such as infection, bleeding, pneumothorax, and vessel puncture, developed in relation to central venous catheter placement.

Lymphocyte removal involves the separation and collection of white blood cells enriched for MNCs from whole blood using centrifugation - based techniques. Due to the proximity of the platelet layer to the MNCs, platelet depletion can be a significant clinical concern (29). Accordingly, patients treated with LPE showed significantly lower platelet counts compared with patients treated with TPE. This suggested that patients with low platelet counts require particular consideration as they may warrant regular monitoring for blood cell count and avoidance of invasive manipulation during the LPE treatment.

The present study included several limitations. First, this was a single-center retrospective study with a small number of LPE patients enrolled. In addition, the criteria for LPE or TPE treatment were based on the decision of the attending physicians. Consequently, selection biases cannot be ruled out. Furthermore, the follow-up period of one year was relatively short and the recurrence of AEs during long-term follow-up remains to be examined. Second, due to constraints of time and cost, this study did not assess whether changes in $\mathrm{B}$ and $\mathrm{T}$ cell numbers, and increased $\mathrm{T}$ suppressor function mediated the improvements in neurological function. Further studies are warranted to further elucidate the mechanisms involved.

Overall, the data demonstrated that both apheresis techniques could efficiently alleviate disability and reduce relapse over a one-year period and is therefore highly feasible for refractory NMOSD patients. Future prospective randomized controlled comparative trials will be beneficial to provide high-quality evidence to evaluate the treatment efficacy of LPE and TPE in NMOSD patients.

\section{Acknowledgments}

The authors thank the physicians and nursing staff in the Department of Blood Transfusion Medicine, the First Medical Center of the Chinese People's Liberation Army General Hospital for their dedication.

Funding: None.

\section{Footnote}

Reporting Checklist: The authors have completed the STROBE reporting checklist. Available at http://dx.doi. org/10.21037/apm-21-177
Data Sharing Statement: Available at http://dx.doi. org/10.21037/apm-21-177

Conflicts of Interest: All authors have completed the ICMJE uniform disclosure form (available at http://dx.doi. org/10.21037/apm-21-177). The authors have no conflicts of interest to declare.

Etbical Statement: The authors are accountable for all aspects of the work in ensuring that questions related to the accuracy or integrity of any part of the work are appropriately investigated and resolved. All procedures performed in this study involving human participants were in accordance with the Declaration of Helsinki (as revised in 2013). This study was been approved by the Ethics Committee of the People's Liberation Army General Hospital. All patients provided signed informed consent for treatment.

Open Access Statement: This is an Open Access article distributed in accordance with the Creative Commons Attribution-NonCommercial-NoDerivs 4.0 International License (CC BY-NC-ND 4.0), which permits the noncommercial replication and distribution of the article with the strict proviso that no changes or edits are made and the original work is properly cited (including links to both the formal publication through the relevant DOI and the license). See: https://creativecommons.org/licenses/by-nc-nd/4.0/.

\section{References}

1. Wingerchuk DM. Neuromyelitis optica: new findings on pathogenesis. Int Rev Neurobiol 2007;79:665-88.

2. Yamamura T, Kleiter I, Fujihara K, et al. Trial of Satralizumab in Neuromyelitis Optica Spectrum Disorder. N Engl J Med 2019;381:2114-24.

3. Kes $\mathrm{P}$, Janssens ME, Bašić-Jukić N, et al. A randomized crossover study comparing membrane and centrifugal therapeutic plasma exchange procedures. Transfusion 2016;56:3065-72.

4. Kaplan AA. Therapeutic plasma exchange: a technical and operational review. J Clin Apher 2013;28:3-10.

5. Morgan SM, Zantek ND, Carpenter AF. Therapeutic plasma exchange in neuromyelitis optica: a case series. J Clin Apher 2014;29:171-7.

6. Bonnan M, Cabre P. Plasma exchange in severe attacks of neuromyelitis optica. Mult Scler Int 2012;2012:787630.

7. Khatri BO, Kramer J, Dukic M, et al. Maintenance plasma 
exchange therapy for steroid-refractory neuromyelitis optica. J Clin Apher 2012;27:183-92.

8. Kumar R, Paul BS, Singh G, et al. Therapeutic Efficacy of Plasma Exchange in Neuromyelitis Optica. Ann Indian Acad Neurol 2018;21:140-3.

9. Jurynczyk M, Messina S, Woodhall MR, et al. Clinical presentation and prognosis in MOG-antibody disease: a UK study. Brain 2017;140:3128-38.

10. Padmanabhan A, Connelly-Smith L, Aqui N, et al. Guidelines on the Use of Therapeutic Apheresis in Clinical Practice - Evidence-Based Approach from the Writing Committee of the American Society for Apheresis: The Eighth Special Issue. J Clin Apher 2019;34:171-354.

11. Pohl M, Kawakami N, Kitic M, et al. T cell-activation in neuromyelitis optica lesions plays a role in their formation. Acta Neuropathol Commun 2013;1:85.

12. Vlasenko AN, Vorob'ev AA, Matveev SIu. Clinical effectiveness of plasmapheresis and lymphocytoplasmapheresis in patients with systemic scleroderma. Klin Med (Mosk) 1992;70:57-61.

13. Liumbruno GM, Centoni PE, Molfettini P, et al. Lymphocytapheresis in the treatment of psoriasis vulgaris. J Clin Apher 2006;21:158-64.

14. Munakata R, Utsugisawa K, Nagane $Y$, et al. No To Shinkei 1999;51:235-9.

15. Luo MC, Wang WF, Yin WF, et al. Clinical efficacy and mechanism of lymphoplasma exchange in the treatment of Guillain-Barre syndrome. Cell Mol Biol (Noisy-le-grand) 2017;63:106-15.

16. Wingerchuk DM, Banwell B, Bennett JL, et al. International consensus diagnostic criteria for neuromyelitis optica spectrum disorders. Neurology 2015;85:177-89.

17. Kurtzke JF. Rating neurologic impairment in multiple sclerosis: an expanded disability status scale (EDSS). Neurology 1983;33:1444-52.

18. Kanamori Y, Nakashima I, Takai Y, et al. Pain in neuromyelitis optica and its effect on quality of life: a cross-sectional study. Neurology 2011;77:652-8.

19. Aungsumart S, Apiwattanakul M. Clinical outcomes and

Cite this article as: Zhang $\mathrm{L}$, Zhuang $\mathrm{Y}$, Liu X, Xu Q, Zhou L, Zou L, Jiang Y, Tian J, Yao H, Chi H, Qiu X, Yang T, Wang $\mathrm{D}, \mathrm{Yu} \mathrm{Y}$. The efficacy of therapeutic apheresis in patients with refractory neuromyelitis optica spectrum disorders: a singlecenter retrospective study. Ann Palliat Med 2021;10(3):3105-3114. doi: 10.21037/apm-21-177 predictive factors related to good outcomes in plasma exchange in severe attack of NMOSD and long extensive transverse myelitis: Case series and review of the literature. Mult Scler Relat Disord 2017;13:93-7.

20. Jiao Y, Cui L, Zhang W, et al. Plasma Exchange for Neuromyelitis Optica Spectrum Disorders in Chinese Patients and Factors Predictive of Short-term Outcome. Clin Ther 2018;40:603-12.

21. Kleiter I, Gahlen A, Borisow N, et al. Apheresis therapies for NMOSD attacks: A retrospective study of 207 therapeutic interventions. Neurol Neuroimmunol Neuroinflamm 2018;5:e504.

22. Iorio R, Pittock SJ. Neuromyelitis optica and the evolving spectrum of autoimmune aquaporin-4 channelopathies. Clin Exp Neuroimmunol 2014;5:175-87.

23. Etemadifar M, Salari M, Mirmosayyeb O, et al. Efficacy and safety of rituximab in neuromyelitis optica: Review of evidence. J Res Med Sci 2017;22:18.

24. Lennon VA, Kryzer TJ, Pittock SJ, et al. IgG marker of optic-spinal multiple sclerosis binds to the aquaporin-4 water channel. J Exp Med 2005;202:473-7.

25. Kim SH, Jeong IH, Hyun JW, et al. Treatment Outcomes with Rituximab in 100 Patients with Neuromyelitis Optica: Influence of FCGR3A Polymorphisms on the Therapeutic Response to Rituximab. JAMA Neurol 2015;72:989-95.

26. Chen B, Wu Q, Ke G, et al. Efficacy and safety of tacrolimus treatment for neuromyelitis optica spectrum disorder. Sci Rep 2017;7:831.

27. Savage WJ, Tobian AA, Savage JH, et al. Scratching the surface of allergic transfusion reactions. Transfusion 2013;53:1361-71.

28. Geiger TL, Howard SC. Acetaminophen and diphenhydramine premedication for allergic and febrile nonhemolytic transfusion reactions: good prophylaxis or bad practice? Transfus Med Rev 2007;21:1-12.

29. Padmanabhan A. Cellular collection by apheresis. Transfusion 2018;58 Suppl 1:598-604.

(English Language Editor: J. Teoh) 